International Journal of Medical Sciences

ISSN 1449-1907 www.medsci.org 2006 3(3):97-105

Research paper

(c)2006 Ivyspring International Publisher. All rights reserved

\title{
Comparison of osteogenic potentials of human rat BMP4 and BMP6 gene therapy using [E1-] and [E1-,E2b-] adenoviral vectors
}

\author{
Hongwei Li ${ }^{1}$, Jin Zhong Li ${ }^{1}$, Debra D. Pittman ${ }^{2}$, Andy Amalfitano ${ }^{3}$, Gerald R. Hankins ${ }^{1}$ and Gregory A. Helm ${ }^{14}$ \\ ${ }^{1}$ Departments of Neurological Surgery, University of Virginia Health System, Charlottesville, Virginia 22908, USA; \\ ${ }^{2}$ Genetics Institute, Andover, Massachusetts 01810, USA; \\ ${ }^{3}$ Departments of Pediatrics and Human Genetics, Duke University Medical Center, Durham, North Carolina 27710, USA; \\ ${ }^{4}$ Departments of Biomedical Engineering, University of Virginia Health System, Charlottesville, Virginia 22908, USA
}

Corresponding address: Jin Zhong Li, D.V.M. Ph.D., Department of Neurological Surgery, University of Virginia Health System, P. O. Box 800212, Charlottesville, Virginia 22908, USA

Received: 2006.05.02; Accepted: 2006.05.31; Published: 2006.06.01

Osteogenic potentials of some recombinant human bone morphogenetic protein (BMP) first-generation adenoviral vectors (ADhBMPs) are significantly limited in immunocompetent animals. It is unclear what role expression of viral proteins and foreign proteins transduced by adenoviral vectors play in the host immune response and in ectopic bone formation. In this study two sets of experiments were designed and performed. First, rat BMP6 cDNA were amplified, sequenced, and recombined in first-generation adenoviral vector (ADrBMP6). A comparison of human and rat BMP6 adenoviral vectors demonstrated identical osteogenic activities in both immunodeficient and immunocompetent rats. Second, the activities of recombinant human BMP6 in E1- (ADhBMP6) and [E1-,E2b-] ([E1-,E2b-]ADGFP\&hBMP6, and [E1-,E2b-]ADhBMP6) adenoviral vectors were compared in both in vitro and in vivo models. Similar activities of these two generations of BMP adenoviral vectors were found in all models. These results indicate that the amount of viral gene expression and the source of the BMP cDNA are not major factors in the interruption of osteogenic potentials of recombinant BMP6 adenoviral vectors in immunocompetent animals.

\section{INTRODUCTION}

Gene therapy provides a novel method to repair damaged bone by using bone morphogenetic protein (BMP). BMP gene vectors can be divided into DNA, viral, and cell vectors $[10,19]$. Among the BMP gene therapy vectors, human BMP adenoviral vectors (ADhBMPs) are very commonly used and have displayed strong osteogenic potentials in immunodeficient animals $[1,25]$. Nevertheless, the functions of ADhBMPs have proved to be significantly limited in immunocompetent animals [1, 25, 35, 38]. All five human BMP adenoviral vectors (ADhBMPs 2, $4,6,7$, and 9) have been shown to induce large volumes of ectopic bone formation in athymic nude (AN) rats. Bone volumes induced by these BMP vectors were greatest when ADhBMPs 4, 6, and 9 were used, followed by ADhBMP2 and, finally, by ADhBMP7. The osteogenic potentials of ADhBMPs 2, 4, and 7, however, were not shown in immunocompetent animals. In addition, bone volumes induced by ADhBMP6 were significantly smaller in immunocompetent animals than in immunodeficient animals. In contrast, bone formation induced by ADhBMP9 was similar in AN and immunocompetent rats [25]. These results may be related to different BMP signal transduction pathways and the host immune response.

The BMP family includes more than 30 members $[9,46]$. According to previous studies, BMPs combine with type 1 (Alk2, Alk3, and Alk6) and type 2 (BR2,
ActR2, and ActR2B) receptors, activate the Smad and p38/MAPK signal transduction pathways, and, finally, activate transcription of bone formation factors [36, 44]. BMP2 and BMP4 combine with Alk3 and use Smad1, Smad5, or Smad8 to transduce signals $[4,31]$. BMP6 and BMP7 may strongly combine with Alk2 and weakly combine with Alk3 and Alk6. Their signals are mainly transferred with Smad5 and, possibly, with Smad1 but not with Smad8 [11, 13]. Compared with other BMPs, BMP9 uses a different type of receptor and signal transduction pathway, the details of which are not yet clear [30, 40]. The functional performances of various BMP adenoviral vectors may reflect different mechanisms in vivo.

The host immune response to an adenovirus and its infected cells may be a primary cause of this functional limitation. The antigenicity of adenoviral vectors, which induce the host immune response, consist of directly injected viral particles and viral genome-expressed proteins that include viral proteins and foreign transgene-encoded proteins [27, 42]. It is unclear what roles expression of viral proteins and foreign proteins transduced by adenoviral vectors play in the host immune response and ectopic bone formation. The adenoviral genome can be divided into four early regions: E1, E2, E3, and E4 [6]. Adenoviral vectors in which the E1 and E3 regions have been deleted have commonly been used in the field of gene therapy and are called first-generation adenoviral vectors, that is, replication-defective adenoviruses. 
Unfortunately, because of the presence of E1-like factors in many cell types, vectors with the E1 deletion may still express a certain amount of viral gene products in vivo. The expression of viral proteins in infected target cells may increase the host immune response and further interrupt the expression of the foreign gene [12]. To limit the productions of potentially immunogenic viral proteins, investigators have pursued the construction of adenoviral vectors in which more or even all viral coding genes have been deleted. Helper-dependent adenoviral vectors in which all viral protein-coding DNA sequences have been deleted have been developed [34, 45]. This modification reportedly has reduced toxicity and prolonged gene expression in some experiments [5, 33]. Nevertheless, we constructed a recombinant BMP9 helper-dependent adenoviral vector and found that the osteogenic potentials of this vector were not significantly different from those of the corresponding recombinant BMP9 first-generation vector (ADhBMP9) in immunodeficient and immunocompetent rats [23]. Because there are different signal transduction pathways among the BMPs, the complex procedure needed to produce the helper-dependent vector and the possible roles of viral gene products in the process of bone formation need to be clarified further. An adenoviral vector with deletions of E1, E3, and the polymerase and terminal protein ( [E1-,E2b-]AD, also known as the second-generation adenoviral vector) was selected in the following study. This class of modified adenovirus vector has several potential benefits including the following: 1) clonal preparations that do not require a helper virus for growth; 2) theoretically, decreased frequency of replication-competent adenovirus generation; 3) increased carrying capacity; 4) rapid scale up of production; and 5) decreased potential for eliciting an immunogenic response in vivo [20]. On the other hand, the sources of BMP cDNAs may also cause the functional limitation of ADhBMPs in immunocompetent animals. We selected rat BMP4 and BMP6 to help us answer these questions.

In the present study, two different sets of experiments were designed and performed. In the first, rat $B M P 6$ cDNA from adult Sprague-Dawley (SD) rats was cloned and sequenced. A recombinant firstgeneration adenovirus ADrBMP6 was constructed and compared with ADhBMP6 in the process of bone formation. In the second set of experiments, human BMP6 cDNA was constructed to form a secondgeneration human BMP adenoviral vector ( [E1-,E2b]ADhBMP). The viral vectors [E1-,E2b-]ADhBMP6, and [E1-,E2b-]ADGFP\&BMP6 which include the green fluorescent protein [GFP] were tested in in vitro and in vivo models. The roles of the viral genome and the sources of BMP cDNA in the process of bone formation were determined in this study.

\section{MATERIALS AND METHODS}

\section{Cloning and identification of rat BMP4 and BMP6 CDNA coding sequences}

Total RNA was prepared from the spleen of a 2month-old SD rat by using an RNeasy Mini Kit (Qiagen, Valencia, $C A$ ) according to the manufacturer's instructions. Rat BMP4 and BMP6 cDNAs containing full coding sequences were generated by performing an RT-PCR with the OneStep RT-PCR Kit (Qiagen). The designs of the PCR primers were based on a known rat BMP4 sequence and a partial rat $B M P 6$ sequence in which mouse and human BMP6 sequences were inserted in places in which the rat sequence was not known [17, 22, 39]. For $B M P 4$, the upstream primer was $5^{\prime}$-CCA CCA TGA TTC CTG GTA ACC GAA TGC TG-3, to which CCACC was added to give the amplified product a typical Kozak consensus sequence around the initiator methionine; the downstream primer was 5'-CTC AGC GGC ATC CGC ACC CCT C-3'. For BMP6, the upstream primer was $5^{\prime}$-TTAGAT CTC CAC CAT GCC CGG GCT GGG G-3', and the downstream primer was 5'-AGA ATC ACA GCC CCT GCA A-3'. Single PCR products of the expected size $(1.2 \mathrm{~kb}$ for $B M P 4$ and $1.5 \mathrm{~kb}$ for BMP6) were purified by performing agarose gel electrophoresis and cloned into the EcoRV site of pShuttleCMV after having been blunted with T4 DNA polymerase. The purified rat $B M P 4$ and BMP6 PCR products and the recombinant plasmids of pShuttle-rBMP4 and pShuttle-rBMP6 were prepared and sequenced (both strands) to ensure that the rat BMP inserts were correct.

\section{Construction of rat BMP4 and BMP6 recombinant adenoviruses}

The AdEasy Vector System [18] was used for construction of the rat BMP4 and BMP6 adenoviral vectors. The linear pShuttle-rBMPs and the pAdEasy 1 plasmid were cotransformed into the competent Escherichia coli strain BJ5183 to obtain the BMP viral DNA plasmid. Briefly, $1 \mu \mathrm{g}$ of linearized recombinant transfer vector pShuttle-rBMP $(5 \mu \mathrm{l})$ and $1.0 \mu \mathrm{l}$ of pAdEasy-1 vector $(100 \mathrm{ng} / \mu \mathrm{l})$ were added to $200 \mu \mathrm{l}$ of competent BJ5183 cells. The next procedure followed methods outlined in previous reports [18]. The recombinant clones were identified by using a PCR.

The pAdeasy- 1 contains the human adenovirus type 5 genome with E1 and E3 deletions. The recombinant adenoviral plasmids, pADrBMP4 and pADrBMP6, were cleaved with PacI to expose their inverted terminal repeats and transfected into 293A cells to produce viral particles. The recombinant viruses were identified by performing PCR, RT-PCR, Southern blot analysis, immunocytochemical staining, and Western blot analysis. The recombinant ADrBMP4 and ADrBMP6 were purified through two cesium chloride gradients, after which the purified virus was desalted by dialysis at $4^{\circ} \mathrm{C}$ against $10 \mathrm{mM}$ Tris-hydrochloric acid buffer with $10 \%$ glycerol and stored in aliquots in liquid nitrogen. The titer of the virus preparations was determined by measuring the 
spectrophotometric absorbance at $260 \mathrm{~nm}$ and by performing a plaque assay.

The AdEasy1 $\Delta \mathrm{pol} \Delta \mathrm{pTp}$ small Vector System ( [E1,E2b-]AD) [3] was used for construction of human BMP second-generation adenoviral vectors ( [E1-,E2b]ADhBMP6, [E1-,E2b-]ADGFP\&hBMP4, and [E1-,E2b]ADGFP\&hBMP6), which lack polymerase and terminal protein [E2b] as well as the E1 and E3 genes. Human BMP4 cDNA was inserted into the shuttle vector pTrackCMV, and a recombinant adenoviral vector was made that encoded both GFP and human BMP4 ( [E1-,E2b-]ADGFP\&hBMP4). Human BMP6 cDNA was inserted into the shuttle vectors pShuttleCMV and pTrackCMV, and recombinant adenoviral vectors were made that encode human BMP6 with GFP ( [E1-,E2b-]ADGFP\&hBMP6) and without GFP ( [E1-,E2b-]ADhBMP6), as described earlier for the AdEasy Vector System. The viruses were produced in C7 cells, which are stably transformed with adenoviral E1 and E2b genes [2]. The viral titer was measured by determining the spectrophotometric absorbance at $260 \mathrm{~nm}$.

\section{Southern blot analysis of genomic DNA of recombinant adenovirus}

Viral DNA was isolated from 293A or C7 cells that had been transduced with recombinant adenovirus. One hundred nanograms of each DNA was digested with HindIII, BstXI (BMP4 and BMP6), or BglII plus EcoRV (BMP6); electrophoretically separated in a $0.8 \%$ agarose gel; and transferred onto a nylon membrane. The membranes were baked at $80^{\circ} \mathrm{C}$ for $30 \mathrm{~min}$ and probed with the pAdEasy1 plasmid, $B M P 4$ cDNA fragment, or BMP6 cDNA fragment, each of which was labeled with digoxigenin by using the DIG-Chem-Link Labeling and Detection Set (Roche Diagnostics Corp., Indianapolis, IN). Detection of DIG-labeled nucleic acids was performed using the DIG Luminescent Detection Kit (Roche).

Western blot detection of BMP4 and BMP6

The pShuttle-rBMP4 and pShuttle-rBMP6 plasmids were transfected into 293A cells with Lipofectamine 2000 reagent (Invitrogen, Carlsbad, CA). African green monkey kidney cells (Vero cells, No. CCL-81; American Type Culture Collection [ATCC], Manassas, VA), which have a higher potential to produce BMPs than 293A or C2C12 cells, were infected with ADrBMP4, ADhBMP4 [25], [E1,E2b-]ADGFP\&hBMP4, ADrBMP6, ADhBMP6 [25], [E1-,E2b-]ADhBMP6, [E1-,E2b-]ADGFP\&hBMP6, or ADNULL $\left(2 \times 10^{8}\right.$ particles per well of a 24-well plate) in Opti-MEMI medium without fetal bovine serum. After transfection, the 293A cells or infected Vero cells were incubated for $48 \mathrm{hrs}$ at $37^{\circ} \mathrm{C}$. The transfected cells and media were harvested, treated in LDS sample buffer (Invitrogen) at $70^{\circ} \mathrm{C}$ for $10 \mathrm{~min}$ with reducing reagent (Invitrogen Corp., Carlsbad, CA), electrophoresed on a NuPAGE $10 \%$ Bis-Tris System (Invitrogen), and transferred to a PVDF membrane (Invitrogen). Recombinant human BMP4 and BMP6 (R\&D Systems, Minneapolis, MN) were used as standard proteins. The blots were reacted with monoclonal mouse BMP4 or BMP6 antibody (Chemicon, Temecula, CA) at $1 \mu \mathrm{g} / \mathrm{ml}$ or with mouse $\beta$-actin antibody (Sigma Chemical Co, St. Louis, MO) at a 1:5000 dilution. The Novex Chemiluminescent Western Blotting Immunodetection System (Invitrogen) was used. Three concentrations of standard BMPs were used to make the standard curves. We calculated the sample BMP concentrations based on the results of film scanning by using the Personal Densitometer SI (Amersham Biosciences, Piscataway, NJ).

\section{Rat BMP4 and BMP6 biological activity assays.}

Mouse C2C12 myoblastic cells (No. CRL-1772; ATCC) were used in bioassays to determine the biological activity of the BMPs. We chose the C2C12 cell line for this experiment not only because increased ALP activity in these cells is dependent on stimulation by BMPs, but also because the $\mathrm{C} 2 \mathrm{C} 12$ cell has a lower background of ALP and constitutes a defined cell line that is much more convenient to use than human mesenchymal stem cells. Cells in 48-well plates that had reached $80 \%$ to $90 \%$ confluence were infected with ADrBMP4, ADhBMP4, ADrBMP6, ADhBMP6, or ADNULL at concentrations of $3 \times 10^{8}, 1.5 \times 10^{8}, 7.5 \times$ $10^{7}$, and $3.8 \times 10^{7}$ particles per well. Seven days later, the cells were stained to measure ALP by using the Sigma Diagnostics ALP Kit (Sigma Diagnostics, Inc., St. Louis, MO).

\section{In vivo ectopic bone formation in rats}

We studied ectopic bone formation in 2-monthold male rats. Eight AN rats and 20 SD rats were used for the study of BMP4, and $16 \mathrm{AN}$ rats and $21 \mathrm{SD}$ rats for the study of BMP6. Animal protocols were approved by the University of Virginia Animal Use and Research Committee and conformed to National Institutes of Health guidelines. The rats were anesthetized with a mixture of ketamine and xylazine, and in each animal the thigh was prepared in a sterile fashion. Using a 19-gauge guide needle, the skin $1 \mathrm{~cm}$ above the knee joint was punctured and the needle advanced $1 \mathrm{~cm}$ proximally. Through this needle, a Hamilton microsyringe was inserted and $50 \mu$ of viral solution $\left(2.8 \times 10^{10}, 5 \times 10^{10}\right.$, or $1.4 \times 10^{11}$ particles $)$ was injected.

For BMP4, the AN rats were separated into two groups (four rats per group). Animals in the first group received an injection of ADrBMP4 in one thigh and an injection of ADNULL in the other. Animals in the other group received an injection of [E1-,E2b]ADGFP\&hBMP4 in one thigh and an injection of ADhBMP4 in the other. Each injection contained $5 \times$ $10^{10}$ particles. The SD rats were separated into four groups ( 5 animals in each group). These animals received bilateral injections $\left(5 \times 10^{10}\right.$ particles in one thigh and $1 \times 10^{10}$ particles in the other) of ADNULL, ADhBMP4, ADrBMP4, or [E1-,E2b-] ADGFP\&hBMP4.

For BMP6, the AN rats were separated into four groups (four rats per group). Animals in three groups received an injection of $2.8 \times 10^{10}$ particles of 
ADNULL in one thigh and an injection of $2.8 \times 10^{10}$ particles of either ADhBMP6, [E1-,E2b]ADGFP\&hBMP6, or [E1-,E2b-]ADhBMP6 in the other thigh. Animals in the remaining group were injected in one thigh with $1.4 \times 10^{11}$ of ADrBMP6 particles and in the other thigh with $2.8 \times 10^{10}$ particles of ADrBMP6. The SD rats were separated into five groups. Only one vector was injected into each SD rat. Animals in the first four groups (four rats per group) received bilateral injections of either ADNULL, ADhBMP6, [E1,E2b-]ADGFP\&hBMP6, or [E1-,E2b-]ADhBMP6 (2.8 × $10^{10}$ particles per thigh). The other five rats received bilateral injections of ADrBMP6 $\left(2.8 \times 10^{10}\right.$ particles in one thigh and $1.4 \times 10^{11}$ particles in the other thigh).

On Day 35, the rats were euthanized and scanned using CT. Axial CT images (1-mm collimation and 1$\mathrm{mm}$ table increment) were obtained using the standard algorithm with $130 \mathrm{kV}, 100 \mathrm{~mA}$, a 2-second scan time, and a 40-mm image size. The threedimensional reconstruction was performed using a Voxel Q workstation. Serum samples were collected on Days 0 and 35.

\section{Detection of antibodies to adenovirus}

Ninety-six-well Nunc Maxisorb plates (Nunc, Inc., Roskilde, Demark) were coated with $100 \mu \mathrm{l}$ of purified adenovirus $\left(5 \times 10^{9}\right.$ particles AdCMV$\beta \mathrm{gal} / \mathrm{ml}$ ) in phosphate-buffered saline (PBS) overnight at $4^{\circ} \mathrm{C}$, washed four times in PBS containing $0.05 \%$ Tween-20, and blocked in PBS supplemented with 1\% bovine serum albumin for $1 \mathrm{hr}$ at $37^{\circ} \mathrm{C}$. Appropriately diluted serum samples were added to antigen-coated plates and incubated overnight at $4{ }^{\circ} \mathrm{C}$. Plates were washed four times in PBS- $0.05 \%$ Tween-20 and incubated with anti-rat IgG $(\mathrm{H}+\mathrm{L})$ ALP conjugate (1:2500 dilution, Promega, Madison, WI) for 2 hrs at $37^{\circ} \mathrm{C}$. The plates were washed in the manner described earlier, and $\rho$-nitrophenyl phosphate $(\rho \mathrm{NPP})$ substrate (Invitrogen) was added. Optical densities were recorded at $410 \mathrm{~nm}$ on an OPTImax tunable microplate reader (Molecular Devices Corp., Sunnyvale, CA).

\section{Detection of antibodies to BMP4 and BMP6}

Microtiter plates (96-well, Nunc) were coated with the purified human BMP4 and BMP6 (R\&D Systems, Minneapolis, MN), followed by blocking, as described earlier. Rat sera were added in serial dilutions. Captured antibodies were detected as described previously.

\section{RESULTS}

Cloning, sequencing, and identification of rat BMP4 and BMP6 cDNAs

Rat BMP4 and BMP6 cDNA fragments containing complete coding regions were amplified by RT-PCR from the total spleen RNA of an adult SD rat. Afterward the fragments were directly cloned into pShuttleCMV, which is a transfer vector used with the CMV promoter for the pAdEasy Vector System.

For rat $B M P 4$ cDNA, five recombinant plasmids were selected and transfected into 293A cells. The expression of BMP4 was determined by performing immunocytochemical staining and Western blot analysis. Three of the five clones produced and secreted BMP4, but the amounts of BMP4 expressed by these clones varied; the other two clones could not produce BMP4 (Fig. 1). The three clones with BMP4 expression were sequenced and their sequences were compared with the BMP4 coding region sequence published in GenBank. Only three consecutive base pairs in the propeptide coding region differed from the rat BMP4 sequence described by GenBank, but these base pairs were the same as those found in murine and human BMP4. One clone that demonstrated a high BMP4 expression was used to construct the rat $B M P 4$ recombinant adenoviral vector. The complete rat BMP4 cDNA sequence was submitted to GenBank (accession No. AY184241).

Figure 1 Western blot detection of rat BMP4 or BMP6 in conditioned media. The conditioned media were prepared from 293A cells transfected with rat BMP4 or BMP6 cDNA recombinant clones. Detection was accomplished using BMP4 antibody (upper panel) and BMP6 antibody (lower panel). Lane 1, pShuttleCMV; Lanes 2-6, rat BMP4 or BMP6 recombinant clones; Lane 7, pShuttle-hBMP4 or pShuttle-hBMP6.

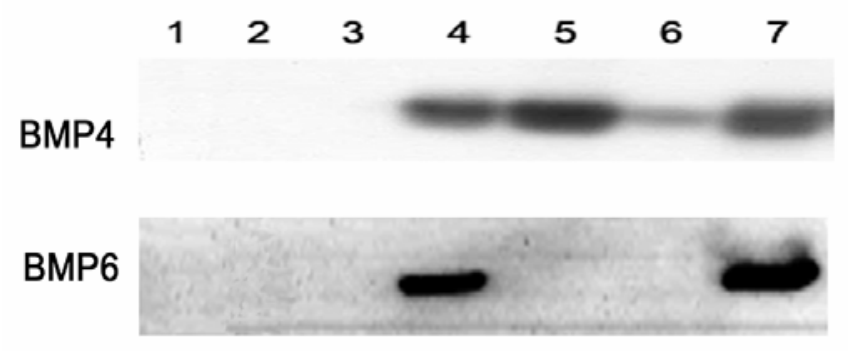

For rat $B M P 6$ cDNA, five recombinant plasmids (Nos. 1, 2, 7, 8, and 23] and the purified PCR product were sequenced. The clones differed from each other and from the partial sequence of the rat BMP6 gene published in GenBank. Only clone No. 8 could produce and secrete BMP6, as observed using immunocytochemical staining and Western blot analysis (Fig. 1). Based on a comparison of the BMP6 sequences of the five clones and the purified PCR product, the first complete rat $B M P 6 \mathrm{cDNA}$ sequence was obtained and submitted to GenBank (accession No. AY184240). For the derived amino acid sequence, amino acids 301 through 506 are identical to those derived from the previously reported partial sequence for the Lewis rat [39] (GenBank accession No. X58830), and amino acids 77 through 280 are identical to those derived from the previously reported partial sequence for the Wistar rat [22] (GenBank accession No. U66298) except for amino acid 146, which in our sequence is valine as opposed to alanine in the Wistar rat. The remaining amino acids, 1 through 76 and 281 through 300 , are identical to those found in the mouse $[8,16$, 17, 28] (GenBank accession No. NM_007556). To 
ensure that the sequence of rat $B M P 6$ cDNA was correct, BMP6 cDNA from another SD rat was amplified, cloned, and sequenced. The findings confirmed that the $B M P 6$ sequences from the two rats were identical. Genomic DNA containing the codon for the polymorphic amino acid was amplified from four additional SD rats and sequenced. The results of the genomic sequencing also verified the presence of valine at position 146 in the SD rats.

In each of the five rat BMP6 clones one or several amino acids were mutated, even though clone No. 8 could produce and secrete BMP6. A rat BMP6 recombinant clone was generated by ligation at the EcoNI site, between the upstream segment of clone No. 8 and the downstream segment of clone No. 2, to obtain a complete clone with no mutated amino acid.

\section{Construction and identification of ADrBMP4 and ADrBMP6}

The AdEasy system was used to construct ADrBMP4 and ADrBMP6. The viral DNA of ADrBMP4 and ADrBMP6 were identified by performing a Southern blot analysis. Figure 2 shows the Southern blot for BMP6. The viral DNA contained the correct rat BMP cDNA inserts, and the expected DNA fragments were generated by using several restriction endonucleases. The titers of the purified viral solutions were $1.0 \times 10^{12}$ particles $/ \mathrm{ml}$ or $2.1 \times$ $10^{10}$ plaque forming units (PFU)/ml for ADrBMP4 and $2.7 \times 10^{12}$ particles $/ \mathrm{ml}$ or $3.3 \times 10^{10} \mathrm{PFU} / \mathrm{ml}$ for ADrBMP6.

Figure 2 Identification of recombinant BMP6 adenoviruses by Southern Blot analysis. A. pAdEasy1 probe. B. BMP6 cDNA fragment probe. DNA samples were digested by HindIII (Lanes 1, 2 and 3), BstXI (Lanes 4, 5, and 6), and BglII plus EcoRV (Lanes 7, 8, and 9). M, 1-kb DNA ladder. Lanes 1, 4, and 7, 293A cells; Lanes 2, 5, and 8, ADrBMP6 DNA; Lanes 3, 6, and 9, ADhBMP6 DNA. This finding indicates that the construction of rat BMP6 recombinant adenovirus is correct.

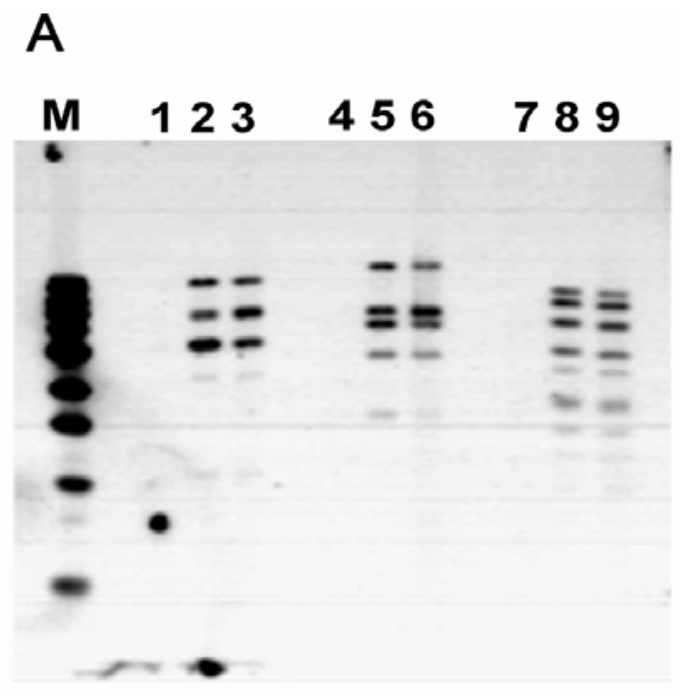

BMP4 and BMP6 expression by corresponding adenoviral vectors in Vero cells

Protein expression by the BMP4 vectors is demonstrated in Fig. 3A and B, which depicts the results of a Western blot analysis of lysed Vero cells transduced with ADNULL, ADhBMP4, or ADrBMP4. The blot was tested with BMP4 antibody (Fig. 3A) and with $\beta$-actin antibody (Fig. 3B). The ADrBMP4transduced cells produced mature protein that exhibited the same electrophoretic mobility as ADhBMP4 on a reduced gel. There was no significant difference in the amount of BMP4 expressed by ADrBMP4- and ADhBMP4-transduced Vero cells. Protein expression by the BMP6 vectors is demonstrated in Fig. 3C, which shows a Western blot of lysed Vero cells transduced with ADNULL, ADhBMP6, or ADrBMP6. The blot was probed with an antibody to BMP6 (Fig. 3C) and with $\beta$-actin antibody (Fig. 3D). The ADrBMP6-transduced cells produced mature protein that exhibited the same electrophoretic mobility as ADhBMP6 on a reduced gel. Also there was no significant difference in the amounts of expressed BMP6 protein between ADrBMP6- and ADhBMP6-transduced Vero cells, which were $8.1 \mu \mathrm{g} / \mathrm{ml}$ and $7.9 \mu \mathrm{g} / \mathrm{ml}$, respectively. After a denaturing gel electrophoresis under reducing conditions and a Western blot analysis, a 19-kD protein reacted with the BMP6 antibody. The staining differed from that of standard human BMP6, which was purchased from R\&D Systems (Minneapolis, MN) and displayed two bands at $18 \mathrm{kD}$ and $23 \mathrm{kD}$. This difference may be due to dissimilar cell types, which may produce different numbers of glycosylation sites.

Alkaline phosphatase activity induced by BMP adenoviral vectors in C2C12 cells

To evaluate the biological function of rat and human BMP4 and BMP6, C2C12 cells transduced with BMP adenoviral vectors were stained to demonstrate

B

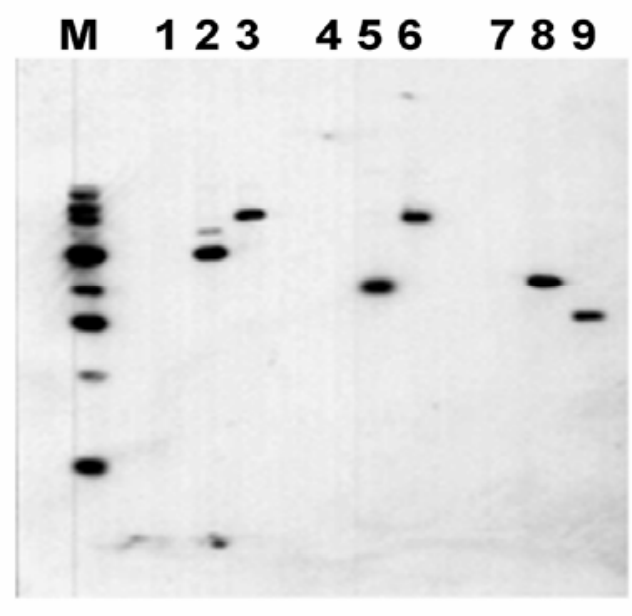

alkaline phosphatase (ALP) activity, which is an important indicator of BMP activity.

The

ADrBMP4induced ALP expression in C2C12 cells was similar to that observed following ADhBMP4 treatment (data not shown); however, at the same number of viral particles, the ALP expression induced by ADrBMP6 in C2C12 cells was significantly 
less than that induced by ADhBMP6 (Fig. 4). No ALP was detected in ADNULL-transduced cells.

Figure 3 Western blot detection of BMP4 (A), BMP6 (C and $E$ ), and $\beta$-actin (B and D). A solution of lyzed transduced Vero cells (A, B, C, and D) or conditioned medium (E) was loaded onto the reduced gel; A. BMP4 antibody detection: Lane 1, Rainbow protein molecular marker; Lane 2, untreated cells; Lane 3, ADNULL; Lane 4, ADhBMP4; Lane 5, ADrBMP4; Lane 6, [E1-,E2b]ADGFP\&hBMP4; Lanes 7, 8 and 9, BMP4 protein standards 20,10, and $5 \mathrm{ng}$. B. The same blot as A tested with $\beta$-actin antibody. C. BMP6 antibody: Lane 1, Rainbow protein molecular marker; Lane 2, untreated cells; Lane 3, ADNULL; Lane 4, ADhBMP6; Lane 5, ADrBMP6; Lanes 6, 7, and 8, BMP6 protein standards 10, 20, and $40 \mathrm{ng}$. D. The same blot as C probed with $\beta$-actin antibody. E. BMP6 antibody detection: Lane 1, Rainbow protein molecular marker; Lane 2, untreated cells; Lane 3, ADNULL; Lane 4, [E1-,E2b-]ADhBMP6; Lane 5, [E1-,E2b-]ADGFP\&hBMP6; Lane 6, ADhBMP6.

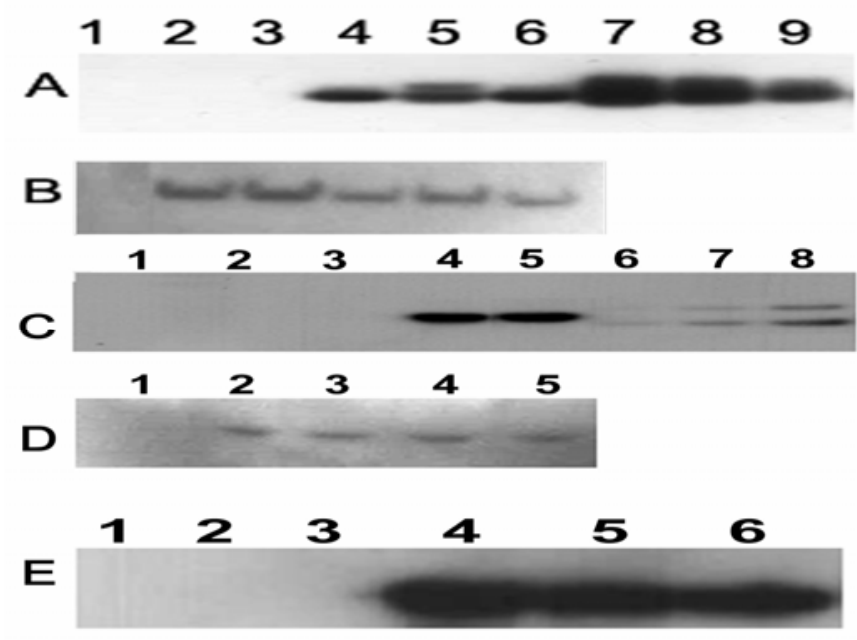

Figure 4 Alkaline phosphatase activity of BMP6 adenoviruses in $\mathrm{C} 2 \mathrm{C} 12$ cells. The $\mathrm{C} 2 \mathrm{C} 12$ cells were transduced with AdCMV-Null, AdCMV-rBMP6 or AdCMV-hBMP6 particles $3 \times 10^{8}$ (A), $1.5 \times 10^{8}$ (B), 7.5x $10^{7}$ (C) and $3.8 \times 10^{7}(\mathrm{D})$.

ADNULL ADhBMP6 ADrBMP6 Particles

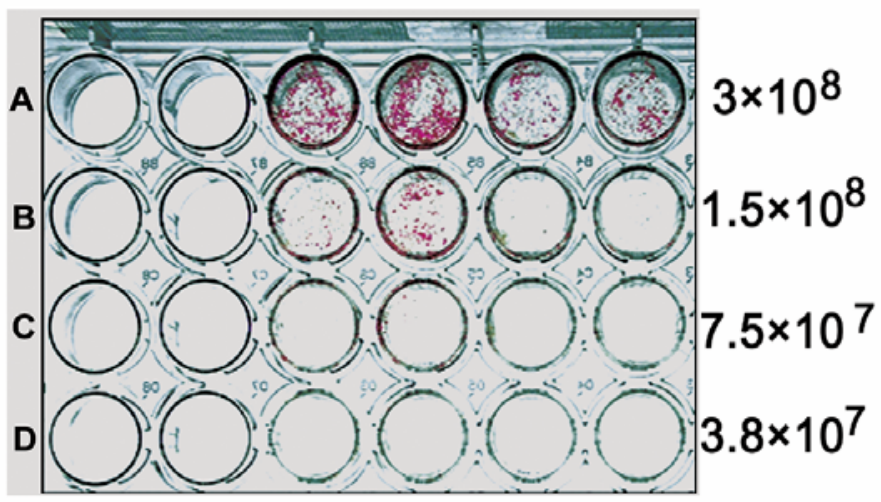

Construction and identification of [E1-, E2b-]ADhBMPs

Human BMP4 and BMP6 cDNAs were also inserted into second-generation adenoviral vectors. These vectors, which included [E1-,E2b]ADGFP\&hBMP4, [E1-,E2b-]ADhBMP6, and [E1-,E2b]ADGFP\&hBMP6, were validated by PCR, RT-PCR, and Southern blot analysis. The titers of the purified [E1-,E2b-]ADGFP\&hBMP4, [E1-,E2b-]ADhBMP6, and [E1-,E2b-]ADGFP\&hBMP6 were $8.6 \times 10^{11}, 9.6 \times 10^{11}$, and $3.6 \times 10^{11}$ particles $/ \mathrm{ml}$, respectively. The amounts of human BMP4 and BMP6 expression in the [E1-,E2b]ADhBMPs were also compared in Vero cells. Similar amounts of mature BMP4 were detected in solutions of lysed cells that had contained [E1-,E2b]ADGFP\&hBMP4 and ADhBMP4 (Fig. 3A and B), and similar amounts of secreted mature BMP6 were detected in cultured media that had contained [E1,E2b-]ADhBMP6, [E1-,E2b-]ADGFP\&hBMP6, and ADhBMP6 (Fig. 3E).

Ectopic bone formation induced by BMP adenoviral vectors

On Day 35 after viral injection, the rats underwent computerized tomography (CT) scanning, and three-dimensional reconstruction was performed using a Voxel $Q$ workstation. The sites at which ADhBMP4, ADrBMP4, and [E1-,E2b-]ADGFP\&hBMP4 had been injected into AN rats revealed similar volumes of ectopic bone formation. None of the BMP4 adenoviruses induced ectopic bone formation at the injection site in SD rats, and ADNULL did not induce ectopic bone formation at the injection site in AN or $\mathrm{SD}$ rats (Fig. 5 upper panel). In AN rats ectopic bone formation was found in the thigh musculature adjacent to the femur, at the injection sites of ADrBMP6 and ADhBMP6. At the same viral dose (2.8 $\times 10^{10}$ particles) the mean volume of bone induced by ADhBMP6 in these animals was larger than that induced by ADrBMP6. Mean bone volumes were greater in AN rats that had received increased viral doses of ADrBMP6 (Table 1). In SD rats, ectopic bone formation was shown at sites that had been injected with $1.4 \times 10^{11}$ particles of ADrBMP6 and 2.8 $\times 10^{10}$ particles of ADhBMP6, but not at the site that had been injected with $2.8 \times 10^{10}$ particles of ADrBMP6. Bone volumes in SD rats that had received one of these two BMP6 vectors were significantly smaller than volumes measured in AN rats that received the same viral dose (Fig. 5 lower panel). Similar patterns of bone formation for [E1-,E2b-]ADhBMP6 and [E1-,E2b]ADGFP\&hBMP6 were observed in both AN and $S D$ rats. 
Figure 5 BMP4- and BMP6-induced bone formation in AN and SD rats. The animals were scanned on Day 35 after viral injection. The viral dose was $5 \times 10^{10}$ particles $/ 50$ $\mu \mathrm{l}$ for all BMP4 vectors (upper panel), $2.8 \times 10^{10}$ particles/50 $\mu$ l for ADhBMP6 and $1.4 \times 10^{11}$ particles/50 $\mu \mathrm{l}$ for ADrBMP6 (lower panel), and $5 \times 10^{10}$ (upper panel) and $1.4 \times 10^{11}$ (lower panel) particles/50 $\mu$ l for ADNULL. Arrows indicate ectopic bone formation.

ADhBMP4 ADrBMP4 [E1-E2b-]ADhBMP4 ADNULL AN
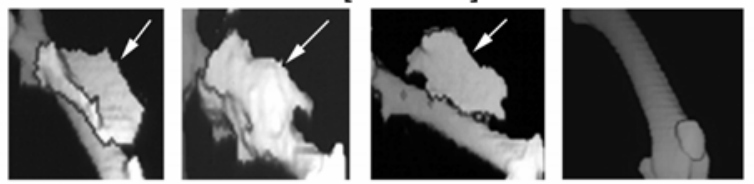

SD
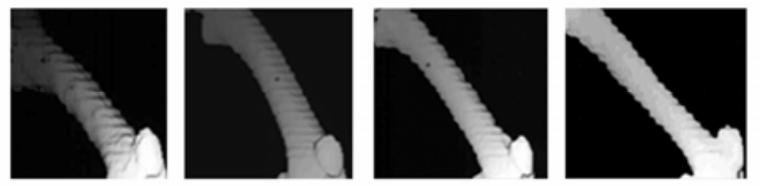

ADhBMP6

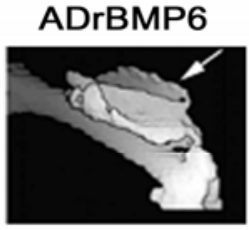

\section{ADNULL}

AN
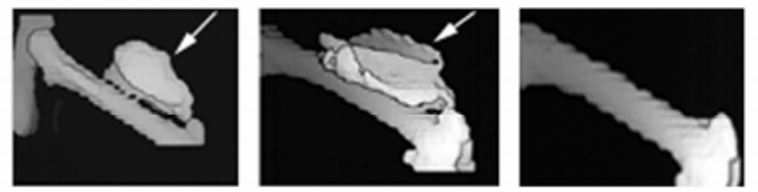

SD
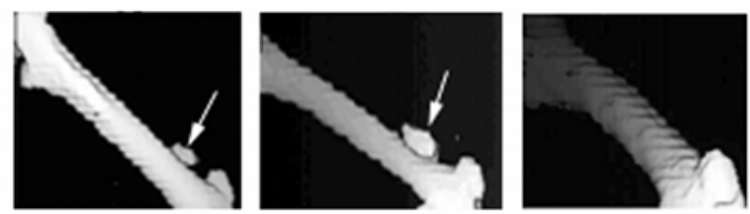

Table 1. Volumes of new bone induced by different BMP6 adenoviruses in AN and SD rats.

\begin{tabular}{|c|c|c|}
\hline Adenovirus & $\begin{array}{c}\text { New Bone in } \\
\text { AN Rats }\left(\mathrm{cm}^{3}\right)\end{array}$ & New Bone in SD Rats $\left(\mathrm{cm}^{3}\right)$ \\
\hline $\begin{array}{c}\text { ADNULL } \\
\left(2.8 \times 10^{10} \text { particles }\right)\end{array}$ & 0 & 0 \\
\hline $\begin{array}{c}\text { ADhBMP6 } \\
\left(2.8 \times 10^{10} \text { particles }\right)\end{array}$ & $1.02 \pm 0.07$ & $0.08 \pm 0.02$ \\
$\begin{array}{c}\text { ADrBMP6 } \\
\left(2.8 \times 10^{10} \text { particles }\right)\end{array}$ & $0.48 \pm 0.09$ & 0 \\
\hline $\begin{array}{c}\text { ADrBMP-6 } \\
\left(1.4 \times 10^{11} \text { particles }\right)\end{array}$ & $1.06 \pm 0.05$ & $0.07 \pm 0.03$ \\
\hline
\end{tabular}

\section{Detection of BMP4 and BMP6 antibodies}

Enzyme-linked immunosorbent assays (ELISAs) were used to analyze sera obtained from the SD rats to determine the presence of antibodies against the adenovirus and against BMP4 and BMP6. Sera from ADNULL-, ADrBMP6-, ADhBMP6 -injected SD rats contained equivalent titers of adenovirus antibodies (Fig. 6A). Sera from ADhBMP6-injected SD rats demonstrated significant antibodies against BMP6, and sera from ADrBMP6-injected rats contained similar titers of antibodies to BMP6 (Fig. 6B). Sera from ADNULL-injected rats contained negligible antibodies to BMP6.

Similarly, sera from ADNULL-, ADhBMP4- and ADrBMP4-injected rats contained equivalent titers of adenovirus antibodies. Sera from ADhBMP4-injected SD rats demonstrated antibodies against BMP4 and, unexpectedly, sera from ADrBMP4-injected SD rats contained similar titers of antibodies to BMP4. Sera from ADNULL-injected SD rats exhibited no reaction to BMP4 (data not shown).

Figure 6 Relative titers of adenovirus and BMP6 antibodies among BMP6 vector- injected SD rats. The SD rat sera were collected on Day 35 after viral injection and tested at a 1:320 dilution by using an ELISA. A. Adenovirus antibody. B. BMP6 antibody.
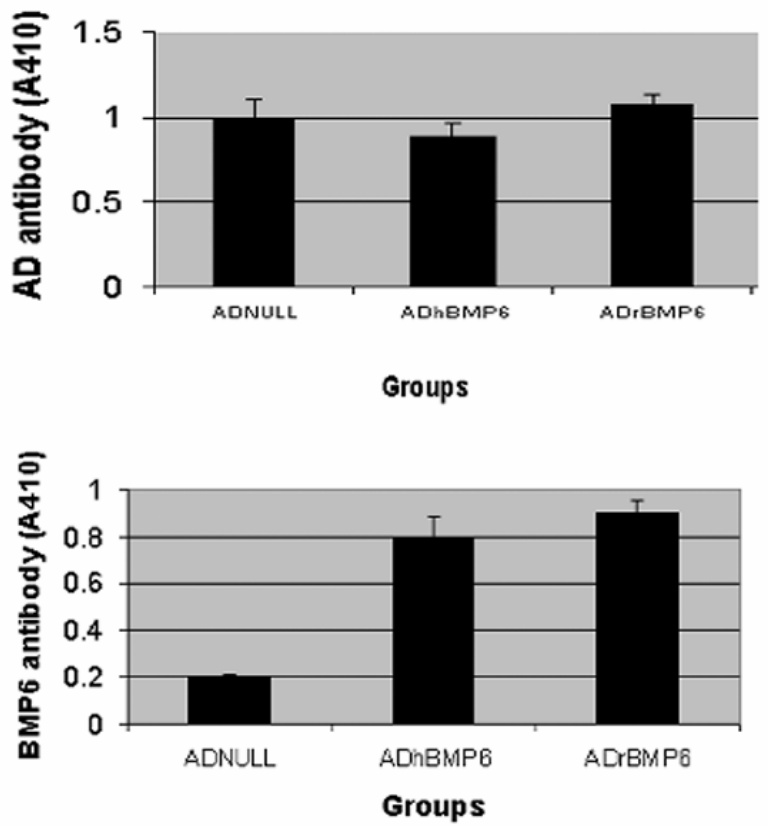

\section{DISCUSIONS}

Although many papers have provided evidence that second- or third-generation adenoviral vectors reduce the host immune response and prolong the expression of transduced foreign genes in immunocompetent animals compared with firstgeneration adenoviral vector, this does not significantly affect the fate of BMP adenoviral vectors in immunocompetent animals. In our previous report [23], we showed that a helper-dependent adenoviral vector did not improve the osteogenic potential of the BMP9 in immunocompetent animals. Because there is a certain amount of helper virus contamination in the preparation of a BMP9 helper-dependent adenovirus and because BMP4, BMP6, and BMP9 have different signal transduction pathways, the effects of viral gene expression in transfected cells on BMP-induced bone formation remained unclear. As a consequence the present study was designed and performed. Recombinant human BMP4 and BMP6 secondgeneration adenoviral vectors have not shown any improvement in the induction of ectopic bone formation in immunocompetent rats. The results of our other experiments demonstrate that foreign gene 
expression curves are very similar in AN and SD rats when a first-generation adenovirus is used. Periods of foreign gene (luciferase) expression can last up to 9 months in SD rats and 6 months in rabbits [24]. The findings indicate that, although similar amounts of BMP4 or BMP6 may be expressed by their adenoviral vectors in $\mathrm{AN}$ and $\mathrm{SD}$ rats, the osteogenic potentials of BMP4 or BMP6 are significantly reduced in immunocompetent rats. This limitation may be caused by the innate host immune response, rather than by the adaptive immune response, which is induced by a direct injection of viral particles [14, 15, 32]. T cells or their secreted cytokines may play critical roles in the process of bone formation.

The source of the foreign genes is another possible factor that may determine the fate of BMP adenoviral vectors. To assess the potential immune response against foreign human $B M P$, rat $B M P 4$ and $B M P 6$ cDNAs were amplified, cloned, sequenced, and identified. Recombinant adenoviruses encoding rat $B M P 4$ and BMP6 were constructed and compared with ADhBMP4 and ADhBMP6 in the in vitro and in vivo models. At the same viral dose, the activities of rat and human BMP4 adenoviral vectors are very similar in all models. Nevertheless, the activity of rat BMP6 is lower than that of human BMP6 in all three models; this is apparent because lower amounts of ALP and smaller volumes of bone were induced by ADrBMP6 than by ADhBMP6 in AN and SD rats. The results indicate that the source of the BMP gene is not a major factor affecting the osteogenic potential of BMP adenoviral vectors in immunocompetent animals. On the other hand, the process of bone formation can be initiated by BMP adenoviral vectors within 1 to 3 days after viral injection [1,21, 23, 43]. This indicates that the early stage after BMP viral injection is the critical period in which to determine the functions of BMP adenoviral vectors in immunocompetent animals. Any additional investigations undertaken to improve the potentials of BMP vectors should target this early stage.

Induction of the host immune response by foreign gene products is a basic immunological principle; however, induction of the host immune response by homologous gene products rarely occurs. In our experiment, BMP4 and BMP6 antibodies in SD rats were detected in the presence of not only ADhBMP4 and ADhBMP6, but also ADrBMP4 and ADrBMP6. It remains unclear why this autoimmune response occurred, but one possible reason may be that local over expression of rat BMPs in muscle tissue provoked the host immune system. Another reason for this autoimmunity may be directly connected to adenoviral vector infection of the muscle cells. It has been shown that adenoviral infections can break the host's tolerance to peripheral transgene-encoded antigens [37, 47], and injection of a foreign protein in adjuvant has been shown to break the host's tolerance to a homologous self protein [26, 29]. It has been demonstrated that adenoviral vectors can also induce immune responses to self-antigens [41]. Interestingly, mice that are transgenic for an E1,E3-deleted adenoviral genome do not appear to tolerate firstgeneration adenoviral vectors, and exposure to adenoviral antigens still elicits the generation of a robust immune response [7]. The overexpression of self-transgenes may, therefore, lead to an autoimmune response and potentially significant side-effects. Thus, human gene therapy trials in which an adenoviral vector containing human genes is utilized should be pursued with caution. Further study of this mechanism may be helpful to understand autoimmune diseases in the human clinical setting.

Besides the aforementioned major findings of this experiment, accurate gene sequencing is also a critical factor in the determination of the fate of gene expression. Any mismatched base pair may lead to the inability of a gene to express its protein. This issue should be emphasized, especially when cDNA is amplified using RT-PCR.

In conclusion, although the deletion of the E1 and $E 2 b$ genes and the use of homologous (rat) BMP genes do not improve the osteogenic potential of direct BMP adenovirus vector gene therapy, the results of this study indicate that BMP gene therapy has unique characteristics and that the initial immune response may play a critical role in the process of bone formation. Interestingly, ADrBMP4 and ADrBMP6 treatments led to the production of anti-self (antirBMP4 or anti-rBMP6) antibodies.

\section{ACKNOLEDGEMENTS}

This study was supported by the National Institutes of Health (Grant No. R01 AR46488-01A2 to GAH). The authors thank John Kammauf for his skills in performing the CT scanning and Dwight Saulle for the genomic DNA PCR on rat BMP6.

\section{CONFLICT OF INTEREST}

The authors have declared that no conflict of interest exists.

\section{REFERENCES}

1. Alden TD, Pittman DD, Hankins GR, et al. In vivo endochondral bone formation using a bone morphogenetic protein 2 adenoviral vector. Hum Gene Ther 1999;10:2245-53.

2. Amalfitano A, Chamberlain JS. Isolation and characterization of packaging cell lines that coexpress the adenovirus E1, DNA polymerase, and preterminal proteins: implications for gene therapy. Gene Ther 1997; 4:258-63.

3. Amalfitano A, Hauser $\mathrm{MA}, \mathrm{Hu} \mathrm{H}$, et al. Production and characterization of improved adenovirus vectors with the E1, E2b, and E3 genes deleted. J Virol 1998; 72:926-33.

4. Aoki H, Fujii M, Imamura T, et al. Synergistic effects of different bone morphogenetic protein type I receptors on alkaline phosphatase induction. J Cell Sci 2001; 114:1483-9.

5. Balague C, Zhou J, Dai Y, et al. Sustained high-level expression of full-length human factor VIII and restoration of clotting activity in hemophilic mice using a minimal adenovirus vector. Blood 2000; 95: 820-8.

6. Berns KI, Giraud C. Adenovirus and adeno-associated virus as vectors for gene therapy. Ann N Y Acad Sci 1995; 772:95-104.

7. Camargo FD, Huey-Louie DA, Finn AV, et al. Germline incorporation of a replication-defective adenoviral vector in 
mice does not alter immune responses to adenoviral vectors. Mol Ther 2000; 2:496-504.

8. Carninci P, Shibata Y, Hayatsu N, et al. Normalization and subtraction of cap-trapper-selected cDNAs to prepare fulllength cDNA libraries for rapid discovery of new genes. Genome Res 2000; 10:1617-30.

9. Ducy P, Karsenty G. The family of bone morphogenetic proteins. Kidney Int 2000; 57:2207-14.

10. Dumont RJ, Dayoub H, Li JZ, et al. Ex vivo bone morphogenetic protein-9 gene therapy using human mesenchymal stem cells induces spinal fusion in rodents. Neurosurgery 2002; 51:12391245.

11. Ebisawa T, Tada K, Kitajima I, Tojo K, Sampath TK, Kawabata $\mathrm{M}$, Miyazono $\mathrm{K}$, Imamura $\mathrm{T}$. Characterization of bone morphogenetic protein-6 signaling pathways in osteoblast differentiation. J Cell Sci 1999; 112:3519-27.

12. Fisher KJ, Choi H, Burda J, et al. Recombinant adenovirus deleted of all viral genes for gene therapy of cystic fibrosis. Virology 1996; 217:11-22.

13. Fujii $M$, Takeda $K$, Imamura $T$, Aoki $H$, et al. Roles of bone morphogenetic protein type I receptors and Smad proteins in osteoblast and chondroblast differentiation. Mol Biol Cell 1999; 10:3801-13.

14. Gahery-Segard H, Farace F, Godfrin D, et al. Immune response to recombinant capsid proteins of adenovirus in humans: antifiber and anti-penton base antibodies have a synergistic effect on neutralizing activity. J Virol 1998; 72:2388-97.

15. Gahery-Segard H, Juillard V, Gaston J, et al. Humoral immune response to the capsid components of recombinant adenoviruses: routes of immunization modulate virus-induced Ig subclass shifts. Eur J Immunol 1997; 27:653-9.

16. Gitelman SE, Kobrin M, Lee A, et al. Structure and sequence of the mouse Bmp6 gene. Mamm Genome 1997; 8:212-4.

17. Gitelman SE, Kobrin MS, Ye JQ, et al. Recombinant Vgr-1/BMP6-expressing tumors induce fibrosis and endochondral bone formation in vivo. J Cell Biol 1994; 126:1595-609.

18. He TC, Zhou S, da Costa LT, et al. A simplified system for generating recombinant adenoviruses. Proc Natl Acad Sci U S A 1998; 95:2509-14.

19. Helm GA, Alden TD, Sheehan JP, Kallmes D. Bone morphogenetic proteins and bone morphogenetic protein gene therapy in neurological surgery: a review. Neurosurgery 2000; 46:1213-22.

20. Hodges BL, Serra D, Hu H, Begy CA, et al. Multiply deleted [E1, polymerase-, and pTP-] adenovirus vector persists despite deletion of the preterminal protein. J Gene Med 2000; 2:250-9.

21. Jane JAJr, Dunford BA, Kron A, et al. Ectopic osteogenesis using adenoviral bone morphogenetic protein (BMP)- 4 and BMP-6 gene transfer. Mol Ther 2002; 6:464-70.

22. Knittel T, Fellmer P, Muller L, Ramadori G. Bone morphogenetic protein- 6 is expressed in nonparenchymal liver cells and upregulated by transforming growth factor-beta 1. Exp Cell Res 1997; 232:263-9.

23. Li JZ, Hankins GR, Kao C, et al. Osteogenesis in rats induced by a novel recombinant helper-dependent bone morphogenetic protein-9 (BMP-9) adenovirus. J Gene Med 2003; 5:748-56.

24. Li JZ, Holman D, Li H, et al. Long-term tracing of adenoviral expression in rat and rabbit using luciferase imaging. J Gene Med 2005;7(6):792-802

25. Li JZ, Li H, Sasaki T, et al. Osteogenic potential of five different recombinant human bone morphogenetic protein adenoviral vectors in the rat. Gene Ther 2003; 10:1735-43.

26. Lin RH, Mamula MJ, Hardin JA, Janeway CAJr. Induction of autoreactive B cells allows priming of autoreactive T cells. J Exp Med 1991; 173:1433-9.

27. Liu Q, Muruve DA. Molecular basis of the inflammatory response to adenovirus vectors. Gene Ther 2003; 10:935-40.
28. Lyons K, Graycar JL, Lee A, et al. Vgr-1, a mammalian gene related to Xenopus $\mathrm{Vg}-1$, is a member of the transforming growth factor beta gene superfamily. Proc Natl Acad Sci U S A 1989; 86:4554-8.

29. Mamula MJ, Lin RH, Janeway CAJr, Hardin JA. Breaking T cell tolerance with foreign and self co-immunogens. A study of autoimmune B and T cell epitopes of cytochrome c. J Immunol 1992; 149:789-95.

30. Miller AF, Harvey SA, Thies RS, Olson MS. Bone morphogenetic protein-9. An autocrine/paracrine cytokine in the liver. J Biol Chem 2000; 275:17937-45.

31. Miyazono K, Kusanagi K, Inoue H. Divergence and convergence of TGF-beta/BMP signaling. J Cell Physiol 2001; 187:265-76.

32. Molinier-Frenkel V, Lengagne $\mathrm{R}$, et al. Adenovirus hexon protein is a potent adjuvant for activation of a cellular immune response. J Virol 2002; 76:127-35.

33. Morral N, O'Neal W, Rice K, Leland M, et al. Administration of helper-dependent adenoviral vectors and sequential delivery of different vector serotype for long-term liver-directed gene transfer in baboons. Proc Natl Acad Sci U S A 1999; 96:12816-21.

34. Morsy MA, Gu M, Motzel S, et al. An adenoviral vector deleted for all viral coding sequences results in enhanced safety and extended expression of a leptin transgene. Proc Natl Acad Sci U S A 1998; 95:7866-71.

35. Musgrave DS, Bosch P, Ghivizzani S, et al. Adenovirusmediated direct gene therapy with bone morphogenetic protein2 produces bone. Bone 1999; 24:541-7.

36. Nohe A, Keating E, Knaus P, Petersen NO. Signal transduction of bone morphogenetic protein receptors. Cell Signal 2004; 16:291-9.

37. Ohashi PS, Oehen S, Buerki K, et al. Ablation of "tolerance" and induction of diabetes by virus infection in viral antigen transgenic mice. Cell 1991; 65:305-17.

38. Okubo Y, Bessho K, Fujimura K, et al. The time course study of osteoinduction by bone morphogenetic protein-2 via adenoviral vector. Life Sci 2001; 70:325-36.

39. Sauermann U, Meyermann R, Schluesener HJ. Cloning of a novel TGF-beta related cytokine, the vgr, from rat brain: cloning of and comparison to homologous human cytokines. J Neurosci Res 1992; 33:142-7.

40. Song JJ, Celeste AJ, Kong FM, et al. Bone morphogenetic protein-9 binds to liver cells and stimulates proliferation. Endocrinology 1995; 136:4293-7.

41. Tripathy SK, Black HB, Goldwasser E, Leiden JM. Immune responses to transgene-encoded proteins limit the stability of gene expression after injection of replication-defective adenovirus vectors. Nat Med 1996; 2:545-50.

42. van Ginkel FW, McGhee JR, Liu C, et al. Adenoviral gene delivery elicits distinct pulmonary-associated $\mathrm{T}$ helper cell responses to the vector and to its transgene. J Immunol 1997; 159:685-93.

43. Varady P, Li JZ, Cunningham M, et al. Morphologic analysis of BMP-9 gene therapy-induced osteogenesis. Hum Gene Ther 2001; 12:697-710.

44. von Bubnoff $\mathrm{A}$, Cho KW. Intracellular BMP signaling regulation in vertebrates: pathway or network? Dev Biol 2001; 239:1-14

45. Von Seggern DJ, Chiu CY, Fleck SK, et al. A helper-independent adenovirus vector with E1, E3, and fiber deleted: structure and infectivity of fiberless particles. J Virol 1999; 73:1601-8

46. Wozney JM. Bone morphogenetic proteins. Prog Growth Factor Res 1989; 1:267-80

47. Zinkernagel RM, Cooper S, Chambers J, et al. Virus-induced autoantibody response to a transgenic viral antigen. Nature 1990; 345:68-71 